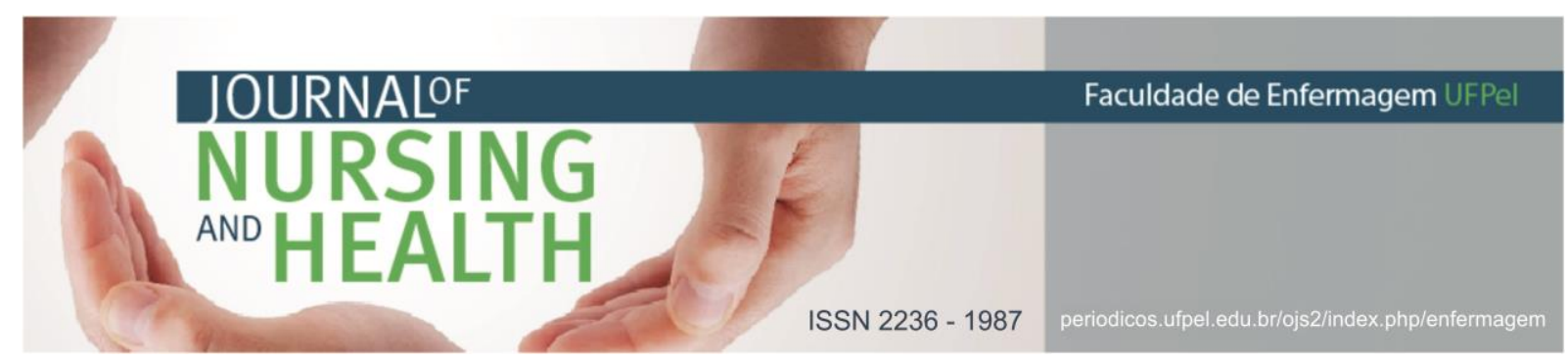

ARTIGO ORIGINAL

\title{
Planejamento reprodutivo de mulheres climatéricas usuárias da Atenção Primária à Saúde Mutirão
}

\author{
Reproductive planning climacteric women users of the Primary Health Care Mutirão \\ Planificación reproductive de las climatericas usuarias de la Atención Primaria de \\ Salud Mutirão
}

Tatiele Estefâni SCHÖNHOLZER ${ }^{1}$, Ione Carvalho PINTO² ${ }^{2}$, Hedi Crecencia Heckler de SIQUEIRA ${ }^{3}$, Queli Lisiane Castro PEREIRA ${ }^{4}$

\section{RESUMO}

Objetivo: conhecer como foi realizado o planejamento reprodutivo das climatéricas usuárias da Atenção Primária em Canarana-MT. Métodos: pesquisa qualitativa, exploratória e descritiva envolvendo dez climatéricas, no ano de 2012, aprovado pelo comitê de ética sob parecer 070/06. Resultados: média de idade foi de 50,2 anos, a média de anos de estudo foi de 7,6. A maioria é casada, sendo 9 sexualmente ativas. São multíparas, variando de 3 a 9 gestações, majoritariamente de alto risco, identificou-se baixo planejamento reprodutivo. $40 \%$ conhecem sobre planejamento familiar. Oito aderiram à laqueadura, minoria reconhece o uso da camisinha para a prevenção de doenças sexualmente transmissíveis. Mesmo sendo inférteis, 40\% identificaram quando interromper a utilização do método contraceptivo. Considerações finais: a assistência ao planejamento reprodutivo contemplou as ações de promoção com êxito, as de prevenção com algumas fragilidades, e ações de informação e educação em saúde de maneira satisfatória ao propiciar conscientização para o autocuidado.

Descritores: Climatério; Planejamento familiar; Saúde da mulher.

\begin{abstract}
Objective: to know how the reproductive planning of climacteric women, users of Primary Health Care in Canarana-MT, was done. Methods: a qualitative, exploratory and descriptive study involving ten climateric, in 2012, approved by the Ethics Committee under protocol 070/06. Results: mean age was 50.2 years, the average years of schooling was 7.6. Most are married, with 9 being sexually active. Are multiparous, ranging from 3 to 9 pregnancies, mostly high-risk, low reproductive planning was identified. $40 \%$ know about family planning. Eight adhered to tubal ligation, few recognize the use of condoms to prevent sexually transmitted diseases. Although being infertile, $40 \%$ identified when to stop using the contraceptive method. Final thoughts: assistance to reproductive planning successfully included promotion actions, prevention with some weaknesses, and information and education on health satisfactorily to provide awareness for self-care.

Descriptors: Climacteric; Family planning; Women's health.
\end{abstract}

\footnotetext{
1 Enfermeira. Doutoranda pelo Programa de Pós-Graduação em Enfermagem em Saúde Pública EERP/USP. Ribeirão Preto, São Paulo, Brasil. E-mail: tatischonholzer@gmail.com

2 Enfermeira. Pós-Doutora. Docente do Departamento de Enfermagem Materno Infantil e Saúde Pública da Escola de Enfermagem de Ribeirão Preto (EERP/USP), São Paulo, Brasil. E-mail: ionecarv@eerp.usp.br

${ }^{3}$ Enfermeira. Doutora em Enfermagem. Professora emérita, titular da Fundação Universidade Federal do Rio Grande no curso de pós-graduação - Mestrado e Doutorado. Líder do Grupo de Estudo e Pesquisa GEES. Rio Grande, RS, Brasil. E-mail: hedihsiqueira@gmail.com

${ }^{4}$ Enfermeira. Doutora. Professora Adjunta do Curso de Bacharelado em Enfermagem do Campus Universitário do Araguaia (CUA) da Universidade Federal de Mato Grosso (UFMT). Membro do Grupo de Estudo e Pesquisa: Gerenciamento Ecossistêmico em Enfermagem/Saúde (GEES). Barra do Garças, Mato Grosso, Brasil. E-mail: quelilisiane@hotmail.com
} 


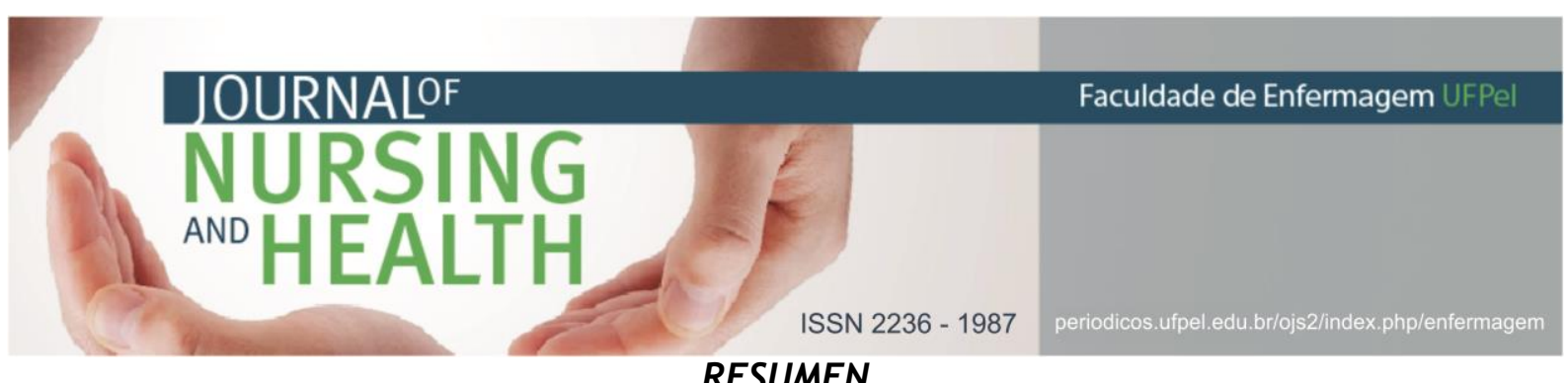

RESUMEN

Objetivo: conocer cómo se realizó la planificación reproductiva de las climatericas en la Atención Primaria en Canarana-MT. Métodos: estudio cualitativo, exploratorio y descriptivo con diez climatericas, en 2012, aprobado por el Comité de Ética, opinión 070/06. Resultados: la edad promedio fue 50,2 años, el promedio de escolaridad fue 7,6. La mayoría son casados, 9 sexualmente activos. Son multíparas, 3 a 9 niños, en su mayoría embarazos de alto riesgo, se identifico baja planificación reproductiva. $40 \%$ sabe acerca de la planificación familiar. Ocho eligieron la ligadura de trompas, pocas reconocen el uso de condones para prevenir las enfermedades de transmisión sexual. Incluso infértiles, $40 \%$ identificó cuando dejar de usar el método anticonceptivo. Consideraciones finales: asistencia a la planificación reproductiva incluyen las acciones de promoción con éxito, la prevención con algunas debilidades, e información y educación sobre la salud de manera satisfactoria proporcionando conciencia para el cuidado personal.

Descriptores: Climaterio; Planificación familiar; Salud de la mujer.

\section{INTRODUÇÃO}

A atenção à saúde sexual e reprodutiva é uma das áreas de atuação prioritárias da Atenção Básica, tem como princípio a garantia dos direitos sexuais e dos direitos reprodutivos, conforme preconizam a Política Nacional de Assistência Integral à Saúde da Mulher. ${ }^{1}$ A assistência ao planejamento reprodutivo contempla ações de promoção, prevenção, informação e educação em saúde. Remete ao Programa de Assistência Integral à Saúde da Mulher (PAISM), que lançado em 1983, inovava com a proposta de cuidado integral à saúde da mulher, não apenas durante 0 período gestacional, e apontava para um planejamento reprodutivo que extrapolasse a oferta e distribuição de contraceptivos. ${ }^{2}$ Abre-se a perspectiva de se considerar as necessidades do indivíduo ao longo da vida, um passo importante para romper com a fragmentação do sujeito. Esses espaços coletivos ou individualizados, de planejamento reprodutivo, devem promover a troca de experiências quanto à vida sexual e reprodutiva da demanda espontânea, programática e reprimida. Sem deixar escapar o seu potencial de promover a autonomia dos usuários para suas escolhas reprodutivas. ${ }^{3-4}$

A fase climatérica, caracterizada pela transição da idade reprodutiva para a idade não reprodutiva, é um período do ciclo vital feminino marcado por alterações, em função da instabilidade hormonal típica da fase pré-menopausal as quais iniciam em média a partir dos 40 anos. Nesta fase de instabilidade podem-se prever os riscos de uma gestação tardia associados ao exercício da vida sexual.5-6 Nos serviços de planejamento familiar há baixa frequência de mulheres no climatério apesar da transição epidemiológica presente $^{7}$, apontando para a importância de serem realizadas ações direcionadas para esse grupo. ${ }^{4}$

Assim, julgamos imperativo conhecer como foi realizado o planejamento reprodutivo das climatéricas usuárias da Atenção Primária em Canarana-MT.

\section{MATERIAIS E MÉTODOS}

Este estudo faz parte do projeto de pesquisa "Processo de Viver da Mulher Climatérica" aprovado pelo 


\section{JOURNAIOF \\ NURSING \\ AND HEALTH}

comitê de ética e pesquisa da Faculdade de Medicina da Universidade Federal de Pelotas-RS sob parecer 070/06, assim sendo, tem sido desenvolvido dentro do referencial da pesquisa qualitativa, privilegiando os discursos dos sujeitos como fonte de informação sem, contudo, restringir a eles a análise. ${ }^{7-11}$ A mesma tem característica exploratória e descritiva.

Foram convidadas a participar da primeira etapa da pesquisa mulheres com idade entre 45 e 55 anos que estavam aguardando atendimento ou acompanhando algum usuário na sala de espera da APS Mutirão do município de Canarana - MT, nos períodos matutinos e vespertinos, de outubro a novembro de 2012. Visando garantir o anonimato dos sujeitos, foram identificados como $\mathrm{C} 1, \mathrm{C} 2, \mathrm{C} 3 . . . \mathrm{Cn}$ conforme a ordem cronológica da realização das entrevistas. Assim, obtiveram-se dez usuárias, as quais contemplaram os seguintes critérios: ser mulher e estar vivenciando o climatério; ter idade entre 45 e 55 anos; ser usuária da APS Mutirão do município de Canarana - MT; ter disponibilidade e interesse de participar da pesquisa; autorizar a gravação da entrevista; assinar o Termo Consentimento Livre Esclarecido; e permitir a divulgação dos dados, respeitando os princípios éticos conforme a resolução CNS 196/96. Foram excluídos do estudo participantes que não se enquadraram nos critérios a cima descritos.

$\begin{array}{crrr}\mathrm{Na} & \text { segunda } & \text { etapa foram } \\ \text { realizadas } & \text { as } & \text { entrevistas }\end{array}$
semiestruturadas com as climatéricas da fase anterior. As entrevistas ocorreram no domicílio das
ISSN 2236 - 1987

climatéricas no mês de novembro de 2012, e eram compostas de questões fechadas e abertas tais como: "A Sra. sabe o que é planejamento familiar? "; "A Sra. utiliza algum método contraceptivo? Por que? "; "A Sra. sabe quando deve cessar o uso? "; "Qual o motivo fez com que a Sra. e o seu esposo/companheiro decidissem pelo método de esterilização cirúrgica? ".

\section{RESULTADO E DISCUSSÃO}

Do total de mulheres que frequentaram a APS em Canarana - MT, que se enquadraram nos critérios de inclusão e aceitaram participar do estudo, totalizaram dez climatéricas. $A$ idade das mulheres foi em média de 50,2 anos, variando de 45 a 55 , sendo a moda de 55 anos.

No que se refere à inserção social, a média de anos frequentados de escola referidos foi de 7,6 anos, variando de um a onze anos, se enquadrando na média da região Centro-Oeste e do país que é de 7,7 anos. $^{8}$

Quanto ao estado civil observouse que oito mulheres são casadas, uma é solteira e uma viúva. Nove climatéricas referiram ser sexualmente ativas, inferindo-se a ausência da síndrome climatérica relacionada à sintomatologia de disfunção sexual (ressecamento vaginal, a dispareunia e a diminuição da libido). Este fato contesta alguns estudos, onde houve presença de disfunção sexual em mais de $30,7 \%$ e $45 \%$, respectivamente, das mulheres climatéricas. ${ }^{9-10}$ Corroborando com os achados deste estudo, pesquisadores afirmam que as mulheres podem manter a libido após a finitude da fase 


\section{JOURNAIOF \\ NURSING \\ AND}

ISSN 2236 - 1987

educação, saúde e alimentação... hoje em dia tem que planejar porque tá tão difícil as coisas (C5).

O tamanho das famílias brasileiras vem diminuindo, média de 1,72 filho por casal, podendo estar relacionado à mudança no papel da família ${ }^{14}$, pois ascensão do feminismo proporcionou melhores oportunidades educacionais e escolhas profissionais mais amplas para as mulheres, postergação da gestação e a constituição de famílias pequenas. ${ }^{15}$

Uma das climatéricas aproximouse do conceito de planejamento familiar, e identificou o êxito do programa de planejamento familiar, como mostram as falas a seguir:

\section{[...] Planejamento familiar é} assim, você planejar a quantidade de filhos que você quer ter. Eu sei o que é planejamento familiar só que assim... a gente nunca teve esse planejamento. Então a gente não tinha aquela orientação igual tem hoje né... e a facilidade é que hoje tem contraceptivo... porque hoje tem no posto de saúde gratuito, engravida só quem quer mesmo... e naquela época a gente não tinha isso né (C7).

A fala supracitada demonstra que compreender, em linhas gerais, para que serve o planejamento familiar não é o suficiente para realizá-lo efetivamente. Para tal, é fundamental ter acesso à educação em saúde, aos insumos a fim de reduzir as iniquidades quanto ao uso dos métodos contraceptivos. A dificuldade relativa ao uso adequado dos métodos tem 


\section{JOURNALOF \\ NURSING \\ MOHEALTH}

colaborado para a ocorrência de gestações não planejadas. ${ }^{12}$ Em seu artigo $4^{\circ}$, a lei 9.263 propõe que o planejamento familiar se oriente por ações preventivas e educativas e pela garantia de acesso igualitário a informações, meios, métodos e técnicas disponíveis para a regulação da fecundidade. 0 planejamento familiar na concepção de C7 auxilia as pessoas que procuram tais serviços, oferecendo-lhes informações necessárias para a escolha e uso efetivo dos métodos anticoncepcionais que melhor se adaptem às condições atuais de saúde, podendo enfim planejar a gestação.

Ao questionar sobre o planejamento familiar na fase climatérica, as pesquisadoras foram surpreendidas pelo fato de que apesar das climatéricas não terem vivenciado a menopausa orgânica, doze meses consecutivos em amenorreia, nenhuma era fecunda, visto que oito realizaram laqueadura tubária, em média, há 22,6 anos e duas, histerectomia. No Brasil, do total de $76,7 \%$ de mulheres em união consensual, $40,0 \%$ são esterilizadas. ${ }^{16}$ Os motivos determinantes para a realização da esterilização cirúrgica, nas climatéricas deste estudo, levou em consideração, o número elevado de filhos, com média de 4,5, variando de três a nove e, as gestações de alto risco, conforme falas a seguir.

\section{[...] o médico perguntou se eu} queria fazer a cirurgia né, pra não ter mais filho porque eu já tinha nove filhos (C1).

A esterilização feminina, no Brasil, é o método contraceptivo mais utilizado 29\%, seguido do anticoncepcional oral $25 \% .^{3}$ Pelo SUS,
ISSN $2236-1987$

no Centro-Oeste no ano de 2010, foram realizados 5.330 procedimentos de laqueadura tubária, destes 33\% foram realizados em Mato Grosso. ${ }^{14}$ A partir de 1996, houve um incremento no quantitativo de esterilização feminina, pois o SUS instituiu a laqueadura como método de planejamento familiar, colocando como indicativo para mulheres acima de vinte e cinco anos, com no mínimo dois filhos vivos, ter acesso a serviço multidisciplinar de aconselhamentos, ter o consentimento do cônjuge, no caso da vigência de união conjugal. ${ }^{1}$ Vale ressaltar que as climatéricas na ocasião da esterilização contemplaram os quesitos à sua realização.

Há conscientização quanto à importância de reduzir os comportamentos de risco e de ter um comportamento sexual responsável, observada nas falas de C9, C10 e C4 ao identificarem a relevância do uso do preservativo masculino na prevenção de doenças sexualmente transmissíveis.

Pra evitar muitas infecções, é pra usar mesmo (C9).

Por eu ter o vírus HPV, porque é o câncer, né... do colo do útero (C10).

Eu acho que é um pouco bobeira da parte da gente...falar: ah to casada, não preciso usar preservativo e tal e vai ficando naquela, entendeu? (C4).

A política para ampliação do acesso ao preservativo masculino no país baseia-se na definição de responsabilidades envolvendo a alocação de recursos financeiros pelas três esferas de gestão. Houve aumento 


\section{JOURNALOF \\ NURSING \\ AND HEALTH}

no acesso e do uso do preservativo masculino na população brasileira. Todavia, a adesão ao preservativo é maior entre as pessoas mais jovens, com primeiro grau completo, e menor entre mulheres, homens negros e na região Centro-Oeste. A adesão mais baixa continua ocorrendo entre os analfabetos. ${ }^{17}$

Desta forma, encontrou-se, no interior do Mato Grosso, climatéricas que apesar dos avanços do Programa Nacional de IST/AIDS, não reconhecem o uso do preservativo como método de prevenção de DST/HIV.

Porque a gente não tinha costume de usar isso (C1).

Porque eu acho que não precisa né (C2).

Não sei porque...é porque...como é que é que eu ia dizer?!... nunca utilizei né... não utilizo (C8).

A ideia de sexo seguro advinda a partir da epidemia da AIDS se integra à perspectiva dos direitos humanos, sendo utilizada como uma forma de atrelar métodos preventivos aos direitos sexuais, que garantem proteção contra doenças e promovem o direito a uma vida sexual satisfatória e segura. No entanto o seu uso está envolto a uma série de psiques ligadas a diminuição do prazer, a estabilidade do relacionamento, a fidelidade entre cônjuges e a falta de conhecimento sobre infecções sexualmente transmissíveis podendo culminar a não adesão do preservativo durante as relações sexuais. ${ }^{18}$

Embora tenham realizado esterilização cirúrgica, laqueadura tubária e histerectomia, quatro
ISSN 2236 - 1987

mulheres, identificaram a finitude da vida reprodutiva ao serem questionadas quando se deve interromper a utilização do método contraceptivo, assim como a fase em que a mulher deixa de ser fértil, como mostram as falas a seguir:

Após a menopausa (C2).

Após a menopausa (C3).

Eu não tenho certeza não, mas acho que a partir dos 45 anos a pessoa não é mais fértil não (C4).

Se a mulher não vai ser mais fértil é quando a mulher fez laqueadura ou se ela entrou na menopausa né (C7).

Pode-se constatar que as usuárias da APS Mutirão têm sido beneficiadas com as ações de educação em saúde pois, como observado nos dados apresentados anteriormente, as mulheres climatéricas souberam identificar o final orgânico da fase reprodutiva. A literatura demonstra que mulheres climatéricas submetidas a intervenções educativas expressam menos crenças negativas sobre 0 climatério e a infertilidade. ${ }^{19}$ Desta forma, considera-se que a abordagem da educação em saúde, é um meio de conscientização das mulheres acerca do seu autoconhecimento e para o autocuidado.

Neste sentido de autoconhecimento e autocuidado, uma das climatéricas reportou a questão de efeitos colaterais clínicos, para interromper 0 uso dos anticoncepcionais, como visto a seguir:

Aah... quando tiver fazendo mal é bom parar né! (C1). 


\section{JOURNALOF \\ NURSING \\ AND HEALTH}

ISSN 2236 - 1987

\section{CONSIDERAÇÕES FINAIS}

Ao conhecer como é realizado o planejamento reprodutivo das climatéricas usuárias da APS Mutirão no município de Canarana-MT pode-se reconhecer que a assistência ao planejamento reprodutivo contemplou as ações de promoção de forma exitosa, as de prevenção com algumas fragilidades, e as ações de informação e educação em saúde de maneira satisfatória ao propiciar conscientização para o autocuidado.

Foi identificada como ação de promoção à saúde a realização das oito laqueaduras tubárias, visto que essas mulheres foram retiradas das condições de risco eminentes das gestações de risco prévias. Já nas ações de prevenção, ancoradas no histórico obstétrico, identificou-se prevalência de gestações não planejadas, evidenciando a fragilidade quanto à eficiência e a adesão às ações do programa de planejamento familiar. Outra fragilidade a ser superada refere-se à necessidade de incentivar maior conscientização quanto à importância de reduzir os comportamentos de risco e de ter um comportamento sexual responsável e assim, reconhecer 0 uso do preservativo na prevenção de infecções sexualmente transmissíveis. Por fim, quanto às ações de informação e educação em saúde, pode-se constatar que as usuárias da APS Mutirão têm sido beneficiadas visto que, algumas climatéricas souberam o quê é o planejamento familiar, o êxito do programa foi identificado, vinculado ao maior acesso à educação em saúde e aos insumos a fim de reduzir as iniquidades quanto ao uso dos métodos contraceptivos. 


\section{NURSING \\ ANO HEALTH}

As ações de educação em saúde também corroboraram, de certa forma, para que as climatéricas se mantivessem sexualmente ativas, não vivenciassem disfunção sexual típica da fase climatérica. Notou-se que a satisfação sexual está em primeiro plano. Também souberam identificar o final orgânico da fase reprodutiva. A educação em saúde é um meio de conscientização das mulheres acerca do seu autoconhecimento e para o autocuidado. Assim, considera-se de suma importância o planejamento reprodutivo na promoção da qualidade de vida das mulheres na fase climatérica.

\section{AGRADECIMENTOS}

A Fundação de Amparo à Pesquisa de Mato Grosso (FAPEMAT) e Conselho Nacional de Desenvolvimento Científico e Tecnológico (CNPq).

\section{REFERÊNCIAS}

1. Ministério da Saúde (BR). Cadernos de Atenção Básica. Saúde sexual e saúde reprodutiva. Brasília; 2013.

2. Pereira QLC. Mulher climatérica usuária do Sistema Único de Saúde: serviços e ações de saúde [dissertação]. Rio Grande do Sul (RS): Universidade Federal do Rio Grande; 2007.

3. Mendonça EAP. Representações médicas e de gênero na promoção da saúde no climatério/menopausa. Ciênc saúde colet. 2004;9(3):751-62.

4. Santos LM, Nakano AR, Bonan C. Planejamento reprodutivo na clínica da família de um Teias: condições facilitadoras e limites à assistência. Saude em debate. 2015;39(106):671-82.
ISSN 2236 - 1987

5. Salazar-Molina A, Klijn TP, Delgado JB. Satisfação sexual nos casais durante o climatério feminino e masculino. Cad saude publica. 2015;31(2):311-20.

6. Brisac JNW, Perin SH, Quayle J. Representação da relação mãe-bebê através do procedimento desenhoestória em gestantes adolescentes e tardias. Mudanças psicol saúde. 2012;19(1-2):69-77.

7. Pereira QLC, Siqueira HCH. Acesso à mamografia: percepções dos responsáveis pela política da saúde da mulher. Reme rev min enferm. 2011;15(3):365-71.

8. Instituto Brasileiro de Geografia e Estatística (IBGE). Taxa de Fecundidade Total - Brasil - 2000 a 2015 [Internet]. 2013 [acesso em 2016 mai 19]. Disponível em: http://brasilemsintese.ibge.gov.br

9. Cavalcanti IF, Farias PDN, Ithamar L, Silva VMD, Lemos A. Função sexual e fatores associados à disfunção sexual em mulheres no climatério. Rev bras ginecol obstet. 2014;36(11):497-502.

10. Nogueira DS. Significados, diferenças ocorridas na pós-menopausa e aspectos positivos relacionados ao climatério em mulheres residentes em Pontal do Araguaia-MT [monografia]. Barra do Garças (MT): Universidade Federal de Mato Grosso; 2011.

11. Pereira QLC, Siqueira HCH. O olhar dos responsáveis pela política de saúde da mulher climatérica. Esc Anna Nery. 2009;13(2):366-71.

12. Evangelista $C B$, Barbieri $M$, Silva PLN. Unplanned pregnancy and the factors associated with the participation in the family planning 


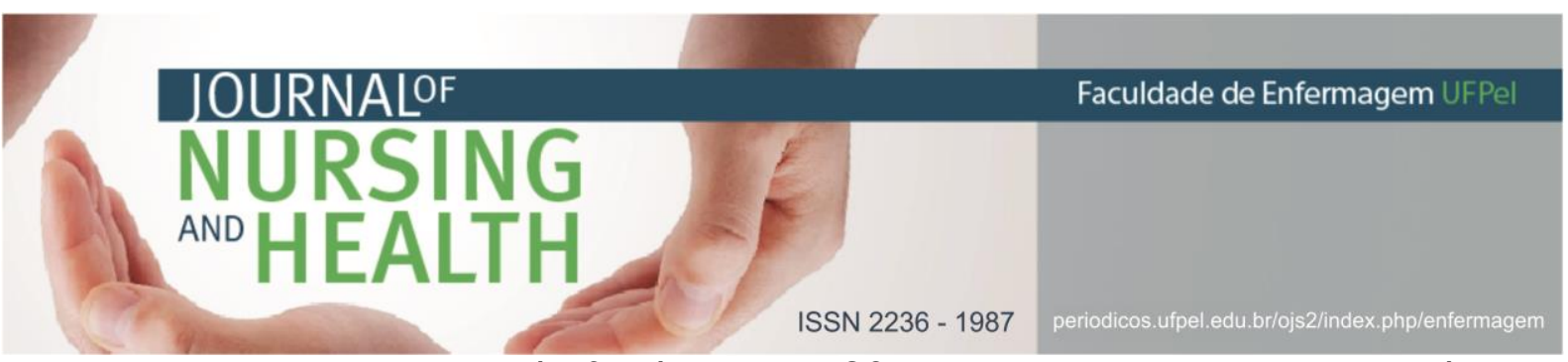

program. Rev pesqui cuid fundam (online). 2015;7(2):2464-74.

20. Arie WMY, Fonseca AM, Melo NR, Bagnoli VR. Anticoncepção no 13. Brasil. Lei $n^{\circ} .9 .263$, de 12 de janeiro de 1996: Regula o $7^{\circ}$ do art. 226 da Constituição Federal, que trata do planejamento familiar, estabelece penalidades e dá outras providências. climatério. RBM: rev bras med. 2004;61(1/2).

Data da submissão: 2016-07-13

Aceito: 2017-03-02

Diário Oficial da União. 15 jan

Publicação: 2017-04-12 1996;Seção 1: 4867-8.

14. Ministério da Saúde (BR). DATASUS. Procedimentos hospitalares do SUS por local de internação [Internet]. 2015 [acesso em 2015 ago 06]. Disponível em: http://tabnet.datasus.gov.br/cgi/tab cgi.exe?sih/cnv/qiuf.def

15. Gravena AAF, Sass A, Marcon SS, Pelloso SM. Resultados perinatais em gestações tardias. Rev esc enferm USP. 2012;46(1):15-21.

16. Caetano AJ. Esterilização cirúrgica feminina no Brasil, 2000 a 2006: aderência à lei de planejamento familiar e demanda frustrada. Rev bras estud popul. 2014;31(2):309-31.

17. Paiva V, Pupo LR, Barboza R. 0 direito à prevenção e os desafios da redução da vulnerabilidade ao HIV no Brasil. Rev saude publica. 2006;40(Suppl):109-19.

18. Pinheiro TF, Calazans GJ, Ayres JRCM. Uso de Camisinha no Brasil: um olhar sobre a produção acadêmica acerca da prevenção de HIV/Aids (2007-2011). Temas psicol. 2013;21(3):815-36.

19. Freitas ER, Barbosa AJG, Reis GA, Remada RF, Moreira LC, Gomes LB, et al. Educação em saúde para mulheres no climatério: impactos na qualidade de vida. Reprod clim. 2016;31(1):3743. 